\title{
Quantum Zeno-based detection and state engineering of ultracold polar molecules
}

\author{
Amit Jamadagni $\odot,{ }^{1, *}$ Silke Ospelkaus, ${ }^{2}$ Luis Santos, ${ }^{1}$ and Hendrik Weimer ${ }^{1}$ \\ ${ }^{1}$ Institut für Theoretische Physik, Leibniz Universität Hannover, Appelstraße 2, 30167 Hannover, Germany \\ ${ }^{2}$ Institut für Quantenoptik, Leibniz Universität Hannover, Welfengarten 1, 30167 Hannover, Germany
}

(Received 21 June 2019; revised 21 July 2021; accepted 17 August 2021; published 2 September 2021)

\begin{abstract}
We present and analyze a toolbox for the controlled manipulation of ultracold polar molecules, consisting of detection of molecules, atom-molecule entanglement, and engineering of dissipative dynamics. Our setup is based on fast chemical reactions between molecules and atoms leading to a quantum Zeno-based collisional blockade in the system. We demonstrate that the experimental parameters for achieving high fidelities can be found using a straightforward numerical optimization. We exemplify our approach for a system composed of $\mathrm{NaK}$ molecules and $\mathrm{Na}$ atoms and we discuss the consequences of residual imperfections such as a finite strength of the quantum Zeno blockade.
\end{abstract}

DOI: 10.1103/PhysRevResearch.3.033208

\section{INTRODUCTION}

Ultracold polar molecules are very promising candidates for a wide range of applications such as quantum simulation [1] or searching for physics beyond the standard model [2]. However, many important techniques for optical control such as optical pumping or cycling transitions for imaging and laser cooling are not available for molecules due to the large number of vibrational levels, presenting a severe obstacle towards realizing these applications. Here, we show that strong chemical reactions between molecules and atoms enable a dissipative interaction mechanism that in the quantum Zeno regime allows one to leverage the optical properties of atoms and achieve the same level of control for the molecules.

In current experiments, ultracold molecules are photoassociated from laser cooled atoms [3,4] with full control over the final hyperfine state [5]. The resulting molecules can be manipulated by external electric fields, microwave driving, or optical potentials, e.g., enabling one to load the molecules into an optical lattice [6,7]. Crucially, ultracold molecules can be subject to chemical reactions, both between themselves as well as with their constituent atoms [8,9]. For some molecules such as $\mathrm{NaK}$ [10-12], the chemical stability with respect to these atom-molecule reactions depends on the atom species involved [13]. With few exceptions [14-16], direct imaging of molecules is only possible in large ensembles and not on the level of a single molecule, as each molecule can scatter only about one photon before ending in a rovibrationally excited state that can no longer scatter additional photons from the imaging laser [17].

\footnotetext{
*amit.jamadagni@itp.uni-hannover.de

Published by the American Physical Society under the terms of the Creative Commons Attribution 4.0 International license. Further distribution of this work must maintain attribution to the author(s) and the published article's title, journal citation, and DOI.
}

In this paper, we present a complete toolbox for transferring the optical control over atoms to the realm of polar molecules. Our approach crucially relies on the presence of strong chemical reactions between atoms and molecules. In the limit where the reaction rate becomes much stronger than the coherent tunneling, the system gets frozen by the quantum Zeno effect, as resonant tunneling becomes unlikely because of the energy uncertainty induced by the dissipative reactions [18]. In this quantum Zeno regime, the dissipative atommolecule interaction implements a collisional blockade that allows for the efficient detection of molecules, overcoming a longstanding challenge. In contrast to previous proposals [19], our approach does not require high-fidelity association and deassociation of the molecules. Furthermore, we demonstrate how to extend our detection method to the generation of atom-molecule entanglement and to the controlled dissipation of rotational excitations of the molecules. We discuss the expected fidelities for experimentally realistic parameters, as well as the role of residual imperfections such as an imperfect quantum Zeno blockade.

In the following, we will first discuss the basic mechanism to implement a detection scheme for the molecules. Later on, we will show how this can be generalized towards the generation of entanglement between the internal degrees of freedom of a molecule and an atom. Finally, we will show how the entangling operation can also be employed towards the controlled dissipation of rotational excitations of the molecule, which can be readily used within laser cooling protocols.

\section{DETECTION OF MOLECULES}

For the detection of the molecules, we consider a bilayer setup of two two-dimensional optical lattice potentials (see Fig. 1). Starting from a dual-Mott insulating state of the two atomic species [20], we first selectively address a single layer of atom pairs for molecule creation by stimulated Raman adiabatic passage (STIRAP). This can be achieved by applying an electric field gradient over the entire lattice resulting in 

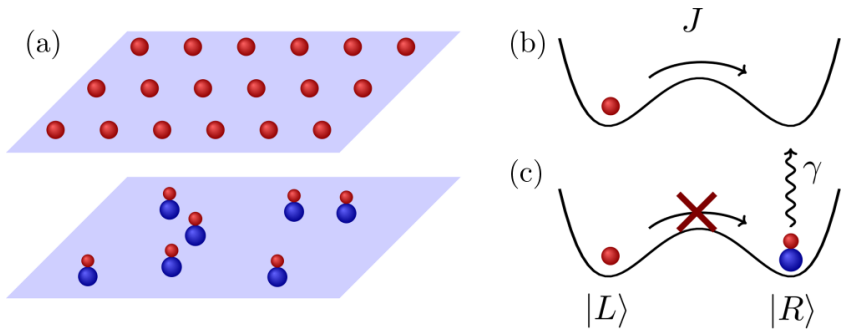

FIG. 1. Quantum Zeno detection of molecules. (a) Bilayer setup of the system with the atoms being in a Mott insulator state in the upper layer separated from the molecules. (b) In the absence of a molecule in the neighboring site, atoms can transfer from $|L\rangle$ to $|R\rangle$. (c) The presence of a molecule inhibits the transfer to $|R\rangle$ due to a strong chemical reaction.

a layer-dependent Stark shift of the rovibrational molecular ground state. The two-photon detuning of the STIRAP lasers can then be tuned to specifically address a single layer of atom pairs for transfer to the rovibrational ground state. This procedure results in a single two-dimensional layer of molecules surrounded by a lattice of atom pairs. In the next step, we apply a resonant light pulse to remove all $\mathrm{K}$ atoms from the entire three-dimensional (3D) lattice. Finally, we apply a magnetic field gradient to transfer a single layer of $\mathrm{Na}$ atoms to a Zeeman state which is dark to subsequently applied removal light. Hidden in this dark state, the gradient is being switched off and a strong removal light pulse removes all remaining particles from the 3D lattice except for the molecular layer and one selected atomic layer of $\mathrm{Na}$ atoms.

For the subsequent analysis, we assume that the tunneling within the two lattice planes is strongly suppressed by the lattice potentials, hence it is sufficient to consider a single double-well setup. Additionally, we consider the atomic layer prepared in a Mott insulator state with one atom per site. Owing to the differences in the AC polarizability [21,22], as well as the fact that the atoms are much lighter than the molecules, we limit the following analysis to the case where only the atom moves with a hopping matrix element $J$, while the molecule remains stationary throughout the process. The crucial dissipative element is provided by a loss with a rate $\gamma$ induced by the chemical reaction between $\mathrm{Na}$ atoms and $\mathrm{NaK}$ molecules $[8,23]$.

The central idea behind the detection mechanism is to attempt a Landau-Zener transfer of the atom to the site potentially occupied by a molecule, e.g., by adiabatically reversing the magnetic field gradient. If there is no molecule on the second site, the transfer will succeed and the atom can be detected using standard quantum gas microscope techniques [24-26]. However, in the presence of molecule in the second site, the strong chemical reaction creates a quantum Zeno blockade [18] of the Landau-Zener process. Hence, detecting the position of the atom after completion of the Landau-Zener sweep allows one to deduce the positions of the molecules.

To be specific, we model the detection in terms of two independent Landau-Zener processes, with and without the presence of a molecule on the second site. For simplicity, we assume half filling of the molecular layer, i.e., equal probabilities for both processes, however the subsequent optimization

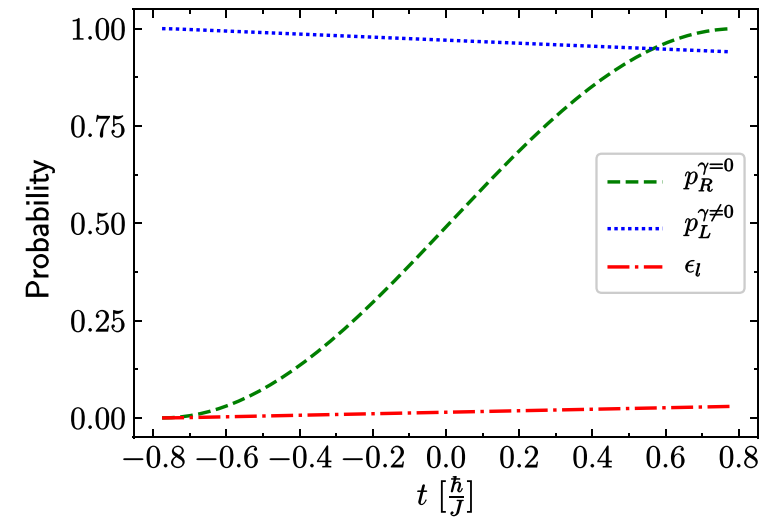

FIG. 2. Atom transfer probability for the atom in the presence $\left(p_{L}^{\gamma \neq 0}\right)$ or absence $\left(p_{R}^{\gamma=0}\right)$ of the molecule. Additionally, the loss $\varepsilon_{l}=$ $1-p_{L}^{\gamma \neq 0}-p_{R}^{\gamma \neq 0}$ is shown $(\gamma=50 \mathrm{~J})$.

can also be tailored towards other molecular densities. Then, the probability for a detection failure (either false positive or false negative) is simply given by the averaged error probability of the two processes. We model the Landau-Zener processes in terms of a non-Hermitian Hamiltonian

$$
H=\Delta(t) \sigma_{z}-J \sigma_{x}-\frac{i \gamma}{2}|R\rangle\langle R|,
$$

where we have used Pauli matrices $\sigma_{\alpha}$ in the basis of the two atom positions $|L\rangle$ and $|R\rangle$, while $\Delta(t)$ describes the influence of the time-dependent magnetic field gradient. The dissipation $\gamma$ vanishes in the absence of a molecule on the right site. The time-dependent term $\Delta(t)$ is taken to be an odd polynomial in $t$ up to fifth order, i.e., satisfying the constraints $\Delta( \pm \infty)=$ $\pm \infty$ and $\Delta(0)=0$. Then, we can minimize the total detection error $\varepsilon$ by optimizing the coefficients of the polynomial as well as the initial time $t_{i}$, while the final time $t_{f}$ is given by $t_{f}=-t_{i}$. As shown in Fig. 2, both Landau-Zener processes can be implemented with high success rate, i.e., the total error $\varepsilon=0.03$ is low, while Fig. 3 captures the optimal $\Delta(t)$ and the error as a function of time with the final time $t_{f}$ optimized. We note that this error can be even further reduced when starting from a high fidelity Mott insulator for the atoms [27], as observing no atom in either site clearly signals the presence of a molecule. This approach can improve the detection fidelity once the preparation fidelity of the Mott insulator surpasses the raw detection fidelity of the molecules.

\section{ATOM-MOLECULE ENTANGLEMENT}

As the next step, we extend the Zeno-based detection scheme towards entanglement of the internal states of atoms and molecules. The internal states $|0\rangle_{a / m}$ and $|1\rangle_{a / m}$ refer to the rovibrational ground state and a rotationally excited state for the molecule $(m)$ and two hyperfine states of the atom $(a)$, respectively. To realize an entangling operation, we consider a setup consisting of three two-dimensional planes, of which we again consider a single three well unit [see Fig. 4(a)]. We constrain the motion of the molecule (atom) to the left (right) site and the center site, respectively. Additionally, we assume that the motion takes place only within the $|1\rangle$ state of both atom and molecule. 
(a)

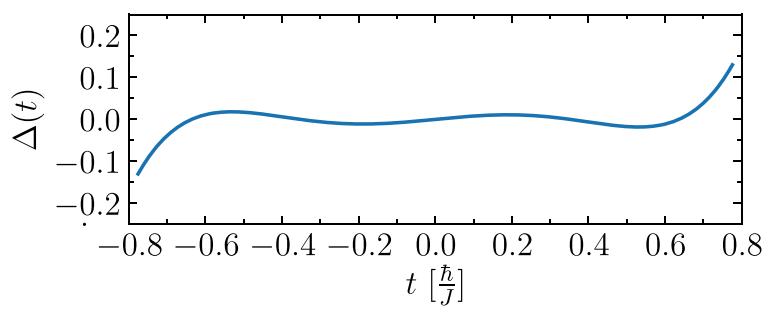

(b)

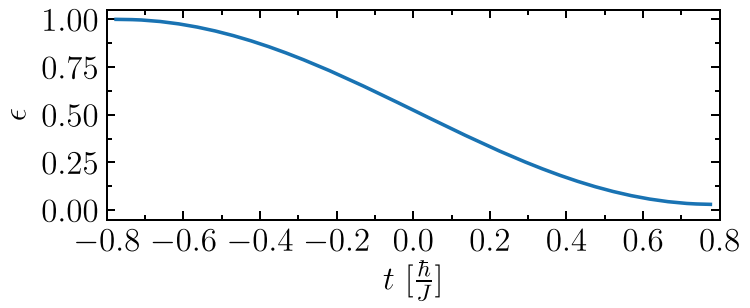

FIG. 3. (a) Optimized profile of the magnetic field gradient $\Delta(t)$. (b) Detection error as a function of time.

A possible scheme to engineer these constrains may be as follows. In addition to the time-dependent magnetic or electric field gradient, the central site $|C\rangle$ may be shifted by an energy difference $\delta$ from that $|L\rangle$ and $|R\rangle$, such that direct hopping is prevented. Sites $|L\rangle$ and $|C\rangle(|R\rangle$ and $|C\rangle)$ are connected along the $x(y)$ direction. We then proceed by connecting the sites via Raman-induced tunneling [28-30]. Crucially, the direction of the Raman beams ensures that hopping is only possible either along the $x$ or the $y$ direction [see Fig. 4(b)], but not around the corner. Specifically, a first Raman pair with frequency transfer $\Delta \omega_{1}=\Delta$, Rabi frequency $\Omega_{1}$, and momentum transfer $\vec{\Delta} k_{1}=\Delta k_{1} \vec{e}_{x}$, induces a molecular hopping rate $J_{m}=$ $\hbar \Omega_{1}\left\langle 1,\left.L\right|_{m} e^{i \Delta k_{1} x} \mid 1, C\right\rangle_{m}$. Crucially, $\left\langle 1,\left.R\right|_{m} e^{i \Delta k_{1} x} \mid 1, C\right\rangle_{m}=0$ due to orthogonality of the Wannier-like functions characterizing each lattice site. As a result, molecules in the $|1\rangle_{m}$ state only hop from $|L\rangle$ to $|C\rangle$. Similarly, using a second Raman pair with $\Delta \omega_{2}=\Delta, \Omega_{2}$, and $\vec{\Delta} k_{2}=\Delta k_{2} \vec{e}_{y}$, we may induce a hopping rate $J_{a}=\hbar \Omega_{2}\left\langle 1,\left.R\right|_{a} e^{i \Delta k_{2} y} \mid 1, C\right\rangle_{a}$, for atoms between $|R\rangle$ and $|C\rangle$ without any atomic hopping from $|C\rangle$ to $|L\rangle$.

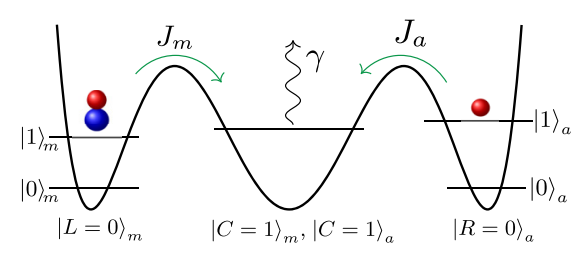

(a)

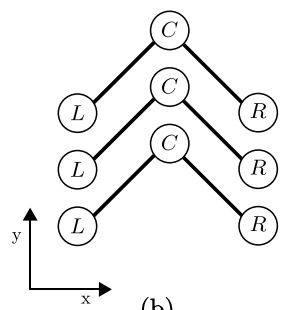

(b)
FIG. 4. Atom-molecule entanglement. (a) Three-well setup for the system with a central reaction site $|C\rangle$, while molecule and atom start in $|L\rangle$ and $|R\rangle$, respectively. (b) The central well which facilitates the chemical reaction is projected out of plane in comparison to the left and the right wells which position the molecule and the atom respectively.

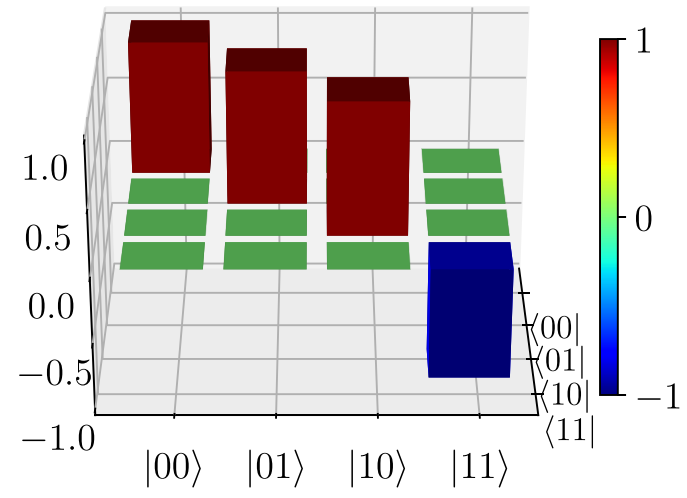

FIG. 5. Matrix representation of the optimized atom-molecule entanglement process, realizing a $\mathrm{CZ}$ gate with fidelity of 0.931 $(\gamma=50 \mathrm{~J})$.

The Hamiltonian of the three-well setup is of the form

$$
\begin{aligned}
H= & B|1\rangle\left\langle\left. 1\right|_{m}+E_{\mathrm{HFS}} \mid 1\right\rangle\left\langle\left. 1\right|_{a}+\Delta_{m}(t) \mid L\right\rangle\left\langle\left. L\right|_{m}\right. \\
& +\Delta_{a}(t)|R\rangle\left\langle\left. R\right|_{a}-\frac{i \gamma}{2} \mid C\right\rangle\left\langle\left. C\right|_{m} \otimes \mid C\right\rangle\left\langle\left. C\right|_{a}\right. \\
& -\left(J_{m}|1, L\rangle\left\langle 1,\left.C\right|_{m}+J_{a} \mid 1, R\right\rangle\left\langle 1,\left.C\right|_{a}+\text { H.c. }\right),\right.
\end{aligned}
$$

where $B$ is the rotational constant of the molecule, $E_{\mathrm{HFS}}$ is the hyperfine splitting of the atom, and $\Delta_{m / a}$ refers to the electric and magnetic field gradients, respectively. For simplicity, we assume the condition $\Delta_{m}(t)=-\Delta_{a}(t) \equiv \Delta(t)$, although this choice is not essential for achieving high fidelity entanglement.

The entangling operation we are realizing corresponds to a controlled- $Z$ (CZ) quantum gate for the internal states. We restrict the computational states to the molecule being in the motional state $|L\rangle$ and the atom being in $|R\rangle$, i.e., any residual occupation of the central site constitutes a gate error. Without loss of generality, we set the tunneling matrix elements to be identical, i.e., $J_{a}=J_{m} \equiv J$. The CZ gate is implemented using a similar Landau-Zener protocol as our detection scheme, however the constraints on $\Delta(t)$ are different. Since the motional degrees of freedom need to return to the initial value at the end of the gate, this requires $\Delta(t)=$ $\Delta(-t)$, therefore we take $\Delta(t)$ to be an even polynomial in $t$ up to fourth order. Then, optimizing the coefficients of the polynomial as well as the gate time results in a close approximation to an exact $\mathrm{CZ}$ gate (see Fig. 5). To capture the performance of the gate in a single number, we use the fidelity $F=\left|\left\langle\psi_{+} \mid \psi\left(t_{f}\right)\right\rangle\right|^{2}$ to create the maximally entangled state $\left|\psi_{+}\right\rangle=\frac{1}{\sqrt{2}}\left(\left|0 L_{m} 0 R_{a}\right\rangle+\left|1 L_{m} 1 R_{a}\right\rangle\right)$, as this constitutes a worst case scenario for a large class of error mechanisms [31]. For a dissipation strength of $\gamma=50 \mathrm{~J}$, we can achieve a fidelity of $F=0.913$. Such a fidelity corresponds to a regime, which allows one to use the atoms as auxiliary particles to mediate interactions between molecules for the digital quantum simulation of important many-body spin models [32]. Another important application of atom-molecule entanglement presents itself after conversion into molecule-molecule entanglement using standard entanglement transfer protocols [33]. Such entangled states of molecules could be readily used in entanglement-assisted precision measurement 


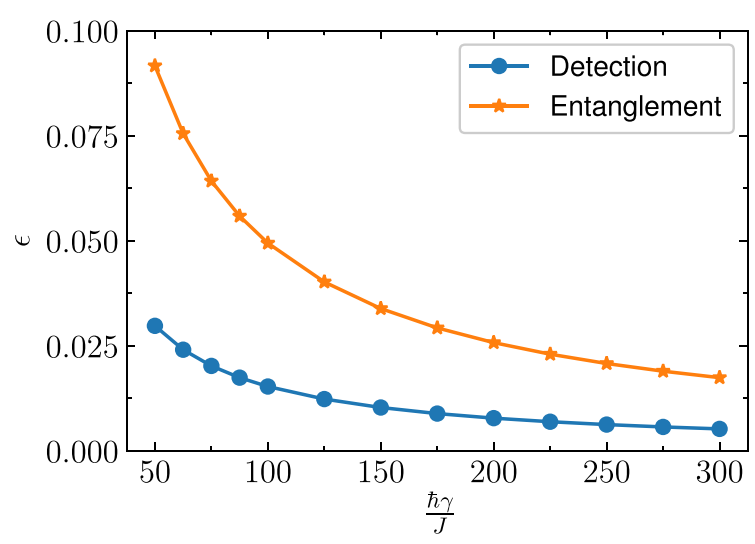

FIG. 6. Errors $\varepsilon$ for the detection and entangling process as a function of $\gamma / J$. The solid lines show fits to quantum Zeno profiles proportional to $1 / \gamma$.

schemes [34], e.g., in searches for a permanent electric dipole moment of the electron [35].

In the previous analyses, we have assumed that the loss rate $\gamma=50 \mathrm{~J}$ is fixed with respect to the tunneling matrix element. However, by tuning the laser parameters for generating the potential wells, it is possible to make this ratio highly tunable. Therefore, it is interesting to investigate how our protocol responds to such a change; in particular there might be practical limitations on how low $J$ can become before other error mechanisms such as thermal losses, buffer gas collisions, etc., kick in. Figure 6 shows that both the detection and the entanglement error $\varepsilon=1-F$ follow the expected $1 / \gamma$ quantum Zeno profile, with the detection error being slightly below the entanglement error.

\section{DISSIPATIVE QUANTUM STATE ENGINEERING}

Let us now extend the mechanism behind the $\mathrm{CZ}$ gate towards the controlled dissipation of rotational excitations. For most polar molecules, such a mechanism cannot reliably be realized using optical pumping due to the large number of vibrational levels an optical excitation can decay into. Nevertheless, such a controlled dissipation channel has immediate application for recoil-free laser cooling of molecules (the recoil is transferred onto the atoms) [36,37], as well as for the dissipative engineering of tailored quantum many-body states [38-40].

To realize this dissipative state engineering, we consider two different strategies. The first strategy is to apply a pulsed scheme, in which the $\mathrm{CZ}$ gate $U_{\mathrm{CZ}}$ is combined with single qubit gates in both the atom and the molecule, in close analogy to a digital quantum simulator. We consider the gate sequence

$$
U=U_{\mathrm{CZ}} R_{x}^{(a)}(\phi) U_{H}^{(m)} U_{\mathrm{CZ}} U_{H}^{(m)} R_{x}^{(a)}(\phi),
$$

where $R_{x}(\phi)=\exp \left(i \phi \sigma_{x}\right)$ is the single qubit rotation about the $x$ axis and $U_{H}$ is the Hadamard gate. After the gate sequence, the molecule and the atom will be entangled. However, a subsequent optical pumping of the atom according to $|1\rangle \rightarrow|0\rangle$ realizes a discrete quantum master equation in Lindblad form according to

$$
\rho_{m}^{\prime}=\rho_{m}+\phi^{2} \sigma_{-}^{(m)} \rho_{m} \sigma_{+}^{(m)}-\frac{\phi^{2}}{2}\left\{\sigma_{+}^{(m)} \sigma_{-}^{(m)}, \rho_{m}\right\}+O\left(\phi^{4}\right) .
$$

This dissipative dynamics can then be interspersed with coherent driving of the rotational states of the molecules to achieve the desired quantum state engineering.

The second strategy for this dissipative state engineering uses a continuous driving of both the molecular and the atomic transitions rather than a pulsed quantum gate sequence. This has two advantages. On the one hand, the mechanism behind the $\mathrm{CZ}$ gate can be understood to implement an effective $\sigma_{z}^{(m)} \sigma_{z}^{(a)}$ interaction, which cannot transfer energy from the molecule to the atom. By going into the rotating frame of the driving, this is converted into a $\sigma_{x}^{(m)} \sigma_{x}^{(a)}$ interaction, which contains terms that lead to an energy transfer. The second advantage is that the energy difference between the rotational constant $B$ and the hyperfine splitting $E_{\mathrm{HFS}}$ is too large to observe direct energy transfer from the molecule to the atom. In the rotating frame, however, this large energy difference is converted into the energy difference involving the respective detunings and Rabi frequencies of the driving. When the differences in the detunings and the Rabi frequencies vanish, efficient energy transfer becomes possible under a condition very similar to the Hartmann-Hahn driving in nuclear magnetic resonance [41]. In this case, the Hamiltonian is given by

$$
\begin{aligned}
H= & -J\left(|1, L\rangle\left\langle 1,\left.C\right|_{m}+\mid 1, R\right\rangle\left\langle 1,\left.C\right|_{a}+\text { H.c. }\right)\right. \\
& +\Omega\left(\sigma_{x}^{(m)}+\sigma_{x}^{(a)}\right)+\delta\left(\sigma_{z}^{(m)}+\sigma_{z}^{(a)}\right) \\
& -\Delta|L\rangle\langle L|-\Delta| R\rangle\langle R|,
\end{aligned}
$$

where $\Omega$ is the Rabi frequency and $\delta$ is the detuning used both for the driving of the molecule and the atom. Additionally, we consider optical pumping of the atom by including a quantum jump operator $c_{\kappa}=\sqrt{\kappa} \sigma_{-}^{(a)}$ in the quantum master equation describing the dynamics:

$$
\frac{d}{d t} \rho=-\frac{i}{\hbar}[H, \rho]+\sum_{i=\gamma, \kappa}\left(c_{i} \rho c_{i}^{\dagger}-\frac{1}{2}\left\{c_{i}^{\dagger} c_{i}, \rho\right\}\right) .
$$

Here, the jump operator $c_{\gamma}$ describes the occurrence of a chemical reaction, which removes both the atom and the molecule from the system. We then optimize the set of parameters $(\Omega, \delta, \Delta, \kappa)$ such that an initial state of $|1\rangle_{m}|0\rangle_{a}$ goes to $|0\rangle_{m}|0\rangle_{a}$. Figure 7 shows that this optimization leads to the initial rotational excitation being dissipated with high probability, while the loss resulting from chemical reaction remains low. We would like to note that this continuous dissipative process might be experimentally easier to realize than a pulsed sequence, however coming at the expense of requiring longer preparation times. We checked the robustness of our protocol with respect to an imperfect experimental implementation of the optimized parameters. Upon decreasing the atomic Rabi frequency by $10 \%$ compared to its optimal value, we observe that the final probability to reach the $|0\rangle_{m}|0\rangle a$ state decreases by merely $2 \%$. This underlines the robustness of our optimization protocol. 


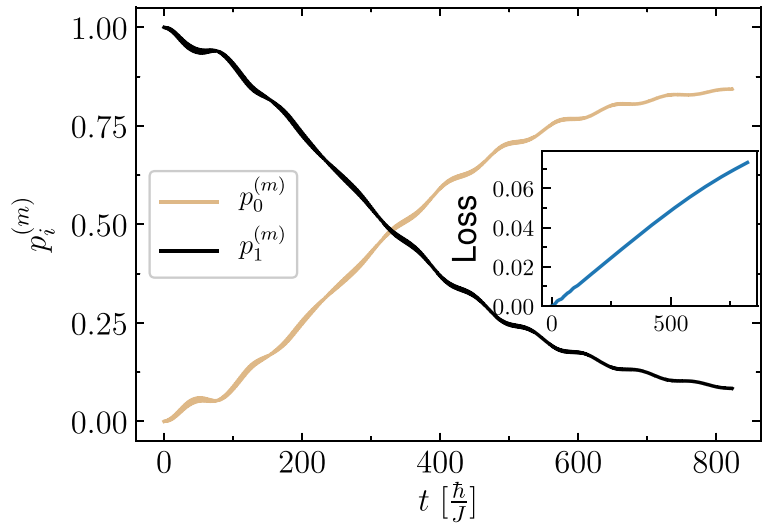

FIG. 7. Dissipation of a rotational excitation. The probability to find the molecule in the rotationally excited state $|1\rangle, p_{1}^{(m)}$ (black) closely follows an exponential decay, while the ground state probability $p_{0}^{(m)}$ increases in a similar way. The inset shows that the overall loss from the chemical reaction remains low $(\gamma=50 \mathrm{~J})$.

\section{EXPERIMENTAL PARAMETERS}

In the following, we will calculate the loss coefficient $\gamma$ for the exothermic reaction $\mathrm{NaK}+\mathrm{Na} \rightarrow \mathrm{Na}_{2}+\mathrm{K}$. The $\mathrm{NaK}+\mathrm{Na}$ system has a van der Waals coefficient of $C_{6}=$ 3592 a.u. [42], from which we can calculate the universal rate coefficient $\beta$ of the chemical reaction [23], which is given by

$$
\beta=\frac{2 \hbar^{3 / 2} \Gamma(1 / 4)^{2}}{\left(2 \mu^{5} C_{6}\right)^{1 / 4}}=1.47 \times 10^{-10} \mathrm{~cm}^{3} \mathrm{~s}^{-1},
$$

where $\mu$ is the reduced mass. The reaction rate $\gamma$ can then be computed as

$$
\gamma=\beta \int d \mathbf{r} n_{\mathrm{at}}(\mathbf{r}) n_{\mathrm{mol}}(\mathbf{r})
$$

where $n_{\text {at }}$ and $n_{\text {mol }}$ refer to the atomic and molecular densities, respectively. In the Gaussian approximation, we obtain

$$
\gamma=\frac{\beta}{8 \pi^{3 / 2} a_{\|} a_{\perp}^{2}},
$$

with $a_{\|}$being the oscillator length along the hopping direction, while $a_{\perp}$ denotes the oscillator length within the molecular layer. In an optical lattice potential corresponding to $a_{\|}=100 \mathrm{~nm}$ and $a_{\perp}=25 \mathrm{~nm}$, this will result in a reaction rate of $\gamma=50 \mathrm{kHz}$. Compared to the typical tunneling matrix element of $J \sim 100-1000 \mathrm{~Hz}$ [43], dissipation rates $\gamma=$ $50-500 \mathrm{~J}$ deep in the quantum Zeno regime can be achieved.

\section{SUMMARY}

In summary, we have presented a toolbox for the controlled manipulation of ultracold polar molecules via dissipative interactions with atoms. Our results pave the way for important experimental advances in the detection, entanglement, and dissipative quantum state preparation of molecular quantum many-body systems. While we have exemplified our approach for $\mathrm{NaK}$, it can also be readily applied to other molecules (including nonpolar ones), provided there is at least one exothermic reaction with one of its constituent atoms. An interesting question for future work is whether the dynamics leading to the formation of ultracold collision complexes [44] or losses from light-assisted collisions [45] can be harnessed for coherent manipulation of molecules in a similar way.

\section{ACKNOWLEDGMENTS}

This work was funded by the Volkswagen Foundation, by the Deutsche Forschungsgemeinschaft (DFG, German Research Foundation) within SFB 1227 (DQ-mat, projects A03 and A04), and under Germany's Excellence Strategy - EXC2123 QuantumFrontiers - 390837967.
[1] S. A. Moses, J. P. Covey, M. T. Miecnikowski, D. S. Jin, and J. Ye, New frontiers for quantum gases of polar molecules, Nat. Phys. 13, 13 (2017).

[2] M. R. Tarbutt, B. E. Sauer, J. J. Hudson, and E. A. Hinds, Design for a fountain of YbF molecules to measure the electron's electric dipole moment, New J. Phys. 15, 053034 (2013).

[3] K.-K. Ni, S. Ospelkaus, M. H. G. de Miranda, A. Pe'er, B. Neyenhuis, J. J. Zirbel, S. Kotochigova, P. S. Julienne, D. S. Jin, and J. Ye, A high phase-space-density gas of polar molecules, Science 322, 231 (2008).

[4] L. De Marco, G. Valtolina, K. Matsuda, W. G. Tobias, J. P. Covey, and J. Ye, A degenerate Fermi gas of polar molecules, Science 363, 853 (2019).

[5] S. Ospelkaus, K.-K. Ni, G. Quéméner, B. Neyenhuis, D. Wang, M. H. G. de Miranda, J. L. Bohn, J. Ye, and D. S. Jin, Controlling the Hyperfine State of Rovibronic Ground-State Polar Molecules, Phys. Rev. Lett. 104, 030402 (2010).

[6] M. H. G. de Miranda, A. Chotia, B. Neyenhuis, D. Wang, G. Quemener, S. Ospelkaus, J. L. Bohn, J. Ye, and D. S. Jin, Controlling the quantum stereodynamics of ultracold bimolecular reactions, Nat. Phys. 7, 502 (2011).
[7] L. Reichsöllner, A. Schindewolf, T. Takekoshi, R. Grimm, and H.-C. Nägerl, Quantum Engineering of a Low-Entropy Gas of Heteronuclear Bosonic Molecules in an Optical Lattice, Phys. Rev. Lett. 118, 073201 (2017).

[8] S. Ospelkaus, K.-K. Ni, D. Wang, M. H. G. de Miranda, B. Neyenhuis, G. Quéméner, P. S. Julienne, J. L. Bohn, D. S. Jin, and J. Ye, Quantum-state controlled chemical reactions of ultracold potassium-rubidium molecules, Science 327, 853 (2010).

[9] M. Guo, X. Ye, J. He, M. L. González-Martínez, R. Vexiau, G. Quéméner, and D. Wang, Dipolar Collisions of Ultracold Ground-State Bosonic Molecules, Phys. Rev. X 8, 041044 (2018).

[10] J. W. Park, S. A. Will, and M. W. Zwierlein, Ultracold Dipolar Gas of Fermionic ${ }^{23} \mathrm{Na}^{40} \mathrm{~K}$ Molecules in Their Absolute Ground State, Phys. Rev. Lett. 114, 205302 (2015).

[11] S. A. Will, J. W. Park, Z. Z. Yan, H. Loh, and M. W. Zwierlein, Coherent Microwave Control of Ultracold ${ }^{23} \mathrm{Na}^{40} \mathrm{~K}$ Molecules, Phys. Rev. Lett. 116, 225306 (2016).

[12] F. Seeßelberg, X.-Y. Luo, M. Li, R. Bause, S. Kotochigova, I. Bloch, and C. Gohle, Extending Rotational Coherence of 
Interacting Polar molecules in a Spin-Decoupled Magic Trap, Phys. Rev. Lett. 121, 253401 (2018).

[13] P. S. Żuchowski and J. M. Hutson, Reactions of ultracold alkalimetal dimers, Phys. Rev. A 81, 060703(R) (2010).

[14] E. S. Shuman, J. F. Barry, and D. Demille, Laser cooling of a diatomic molecule, Nature (London) 467, 820 (2010).

[15] M. T. Hummon, M. Yeo, B. K. Stuhl, A. L. Collopy, Y. Xia, and J. Ye, 2D Magneto-Optical Trapping of Diatomic Molecules, Phys. Rev. Lett. 110, 143001 (2013).

[16] T. A. Isaev and R. Berger, Polyatomic Candidates for Cooling of Molecules with Lasers from Simple Theoretical Concepts, Phys. Rev. Lett. 116, 063006 (2016).

[17] D. Wang, B. Neyenhuis, M. H. G. de Miranda, K.-K. Ni, S. Ospelkaus, D. S. Jin, and J. Ye, Direct absorption imaging of ultracold polar molecules, Phys. Rev. A 81, 061404(R) (2010).

[18] B. Zhu, B. Gadway, M. Foss-Feig, J. Schachenmayer, M. L. Wall, K. R. A. Hazzard, B. Yan, S. A. Moses, J. P. Covey, D. S. Jin, J. Ye, M. Holland, and A. M. Rey, Suppressing the Loss of Ultracold Molecules Via the Continuous Quantum Zeno Effect, Phys. Rev. Lett. 112, 070404 (2014).

[19] J. P. Covey, L. D. Marco, Ó. L. Acevedo, A. M. Rey, and J. Ye, An approach to spin-resolved molecular gas microscopy, New J. Phys. 20, 043031 (2018).

[20] S. Sugawa, K. Inaba, S. Taie, R. Yamazaki, M. Yamashita, and Y. Takahashi, Interaction and filling-induced quantum phases of dual Mott insulators of bosons and fermions, Nat. Phys. 7, 642 (2011).

[21] W. F. Holmgren, M. C. Revelle, V. P. A. Lonij, and A. D. Cronin, Absolute and ratio measurements of the polarizability of $\mathrm{Na}, \mathrm{K}$, and $\mathrm{Rb}$ with an atom interferometer, Phys. Rev. A 81, 053607 (2010).

[22] B. Neyenhuis, B. Yan, S. A. Moses, J. P. Covey, A. Chotia, A. Petrov, S. Kotochigova, J. Ye, and D. S. Jin, Anisotropic Polarizability of Ultracold Polar ${ }^{40} \mathrm{~K}{ }^{87} \mathrm{Rb}$ Molecules, Phys. Rev. Lett. 109, 230403 (2012).

[23] Z. Idziaszek and P. S. Julienne, Universal Rate Constants for Reactive Collisions of Ultracold Molecules, Phys. Rev. Lett. 104, 113202 (2010).

[24] W. S. Bakr, J. I. Gillen, A. Peng, S. Fölling, and M. Greiner, A quantum gas microscope for detecting single atoms in a Hubbard-regime optical lattice, Nature (London) 462, 74 (2009).

[25] J. F. Sherson, C. Weitenberg, M. Endres, M. Cheneau, I. Bloch, and S. Kuhr, Single-atom-resolved fluorescence imaging of an atomic Mott insulator, Nature (London) 467, 68 (2010).

[26] E. Haller, J. Hudson, A. Kelly, D. A. Cotta, B. Peaudecerf, G. D. Bruce, and S. Kuhr, Single-atom imaging of fermions in a quantum-gas microscope, Nat. Phys. 11, 738 (2015).

[27] W. S. Bakr, P. M. Preiss, M. E. Tai, R. Ma, J. Simon, and M. Greiner, Orbital excitation blockade and algorithmic cooling in quantum gases, Nature (London) 480, 500 (2011).

[28] D. Jaksch and P. Zoller, Creation of effective magnetic fields in optical lattices: the Hofstadter butterfly for cold neutral atoms, New J. Phys. 5, 56 (2003).
[29] M. Aidelsburger, M. Atala, S. Nascimbène, S. Trotzky, Y.-A. Chen, and I. Bloch, Experimental Realization of Strong Effective Magnetic Fields in an Optical Lattice, Phys. Rev. Lett. 107, 255301 (2011).

[30] H. Miyake, G. A. Siviloglou, C. J. Kennedy, W. C. Burton, and W. Ketterle, Realizing the Harper Hamiltonian with LaserAssisted Tunneling in Optical Lattices, Phys. Rev. Lett. 111, 185302 (2013).

[31] H. Weimer, N. Y. Yao, C. R. Laumann, and M. D. Lukin, LongRange Quantum Gates using Dipolar Crystals, Phys. Rev. Lett. 108, 100501 (2012).

[32] H. Weimer, Quantum simulation of many-body spin interactions with ultracold polar molecules, Mol. Phys. 111, 1753 (2013).

[33] A. Barenco, C. H. Bennett, R. Cleve, D. P. DiVincenzo, N Margolus, P. Shor, T. Sleator, J. A. Smolin, and H. Weinfurter, Elementary gates for quantum computation, Phys. Rev. A 52, 3457 (1995).

[34] D. Leibfried, M. D. Barrett, T. Schaetz, J. Britton, J. Chiaverini, W. M. Itano, J. D. Jost, C. Langer, and D. J. Wineland, Toward Heisenberg-limited spectroscopy with multiparticle entangled states, Science 304, 1476 (2004).

[35] V. Andreev, D. G. Ang, D. DeMille, J. M. Doyle, G. Gabrielse, J. Haefner, N. R. Hutzler, Z. Lasner, C. Meisenhelder, B. R. O'Leary, C. D. Panda, A. D. West, E. P. West, X. Wu, and A. Collaboration, Improved limit on the electric dipole moment of the electron, Nature (London) 562, 355 (2018).

[36] S. D. Huber and H. P. Büchler, Dipole-Interaction-Mediated Laser Cooling of Polar Molecules to Ultracold Temperatures, Phys. Rev. Lett. 108, 193006 (2012).

[37] B. Zhao, A. W. Glaetzle, G. Pupillo, and P. Zoller, Atomic Rydberg Reservoirs for Polar Molecules, Phys. Rev. Lett. 108, 193007 (2012).

[38] S. Diehl, A. Micheli, A. Kantian, B. Kraus, H. P. Büchler, and P. Zoller, Quantum states and phases in driven open quantum systems with cold atoms, Nat. Phys. 4, 878 (2008).

[39] F. Verstraete, M. M. Wolf, and J. Ignacio Cirac, Quantum computation and quantum-state engineering driven by dissipation, Nat. Phys. 5, 633 (2009).

[40] H. Weimer, M. Müller, I. Lesanovsky, P. Zoller, and H. P. Büchler, A Rydberg quantum simulator, Nat. Phys. 6, 382 (2010).

[41] S. R. Hartmann and E. L. Hahn, Nuclear double resonance in the rotating frame, Phys. Rev. 128, 2042 (1962).

[42] P. S. Żuchowski, M. Kosicki, M. Kodrycka, and P. Soldán, van der Waals coefficients for systems with ultracold polar alkalimetal molecules, Phys. Rev. A 87, 022706 (2013).

[43] I. Bloch, J. Dalibard, and W. Zwerger, Many-body physics with ultracold gases, Rev. Mod. Phys. 80, 885 (2008).

[44] M. Mayle, B. P. Ruzic, and J. L. Bohn, Statistical aspects of ultracold resonant scattering, Phys. Rev. A 85, 062712 (2012).

[45] A. Christianen, M. W. Zwierlein, G. C. Groenenboom, and T. Karman, Photoinduced Two-Body Loss of Ultracold Molecules, Phys. Rev. Lett. 123, 123402 (2019). 